\title{
ABSTRACT CELLULARIZATION AS A CELLULARIZATION WITH RESPECT TO A SET OF OBJECTS
}

\author{
BORIS CHORNY
}

\begin{abstract}
Given a simplicial idempotent augmented endofunctor $F$ on a simplicial combinatorial model category $\mathcal{N}$, under the assumption of Vopernka's principle, we exhibit a set $A$ of cofibrant objects in $\mathcal{M}$ such that $F$ is equivalent to $\mathrm{CW}_{A}$, the cellularization with respect to $A$.
\end{abstract}

\section{INTRODUCTION}

Over the past ten years many results about locally presentable categories were generalized to combinatorial model categories. The term "combinatorial" means that the model category is cofibrantly generated and the underlying category is locally presentable (see [1] and [10 for the definitions of these concepts). This notion is due to J. H. Smith, who constructed (in unpublished work) localizations of combinatorial model category structures with respect to sets of maps. This construction may be viewed as a generalization of the orthogonal reflection in a locally presentable model category [1, 1.35] and the claim that every small-orthogonality class of a locally presentable category is locally presentable [1 1.40]. In [9], Dugger proved that every combinatorial model category is equivalent to a localization of a category of diagrams of simplicial sets, hence generalizing [1 1.46]. Furthermore, J. Rosický have recently proved (personal communication) that for strongly combinatorial model category $\mathcal{K}$, (cone) injectivity classes in the homotopy category of $\mathcal{K}$ behave similarly as in locally presentable categories. $\mathcal{K}$ is strongly combinatorial if it is combinatorial and the class of cofibrations is closed under $\kappa$-directed colimits in $\operatorname{Map}(\mathcal{K})$ for some regular cardinal $\kappa$.

Vopěnka's principle has especially powerful implications for locally presentable categories if one is ready to assume it [1, Chapter 6]. In particular existence of localization with respect to an arbitrary class of maps in a locally presentable category is equivalent to the weak Vopěnka's principle. Substituting weak Vopěnka's principle by a stronger Vopěnka's principle, J.Rosický and W. Tholen 11] proved an analogous result for combinatorial model categories: Vopěnka's principle implies the existence of homotopical localizations in combinatorial model categories with respect to arbitrary classes of maps. In the category of simplicial sets this result was proven earlier in the work of C. Casacuberta, D. Scevenels, and J. Smith [5].

If Vopěnka's principle is assumed, a stronger statement for locally presentable categories holds: any orthogonality class is a small orthogonality class (i.e., it is generated by a set of maps). An analog for combinatorial model categories is the

Date: July 6, 2018.

1991 Mathematics Subject Classification. Primary 55U35; Secondary 55P91, 18 G55.

Key words and phrases. model category, cellularization, large cardinal. 
following: any homotopy localization functor is a localization with respect to a set of maps [2]. There exists a counterexample for this statement in a locally presentable category which is not cofibrantly generated [7, so combinatorial model categories are the right generalization of locally presentable model categories.

We will finish this comparison of locally presentable categories with combinatorial model categories with one example of a statement which was not generalized yet: any orthogonality class generated by a set of objects in a complete and wellpowered category was shown to be reflective in [3]. An analogous statement in topology is known better as a problem of existance of homotopical localization with respect to a cohomology theory. Cohomological localizations are known to exist under Vopěnka's principle, but in ZFC this problem is still open.

In this paper we continue the efforts of transferring results from locally presentable category theory to abstract homotopy theory. Namely we generalize the following statement: assuming Vopěnka's principle any co-orthogonality class in a locally presentable category is co-reflective [1, 6.29]. We show that under Vopěnka's principle any homotopy co-orthogonality class in a combinatorial model category may be generated by a set of objects and hence (if the category is right proper) admits a homotopy co-localization (e.g., by [10, Prop. 5.3.5] applied to a map $\emptyset \rightarrow X)$.

Next we point out that any cellularization (homotopy co-localization) in a simplicial combinatorial model category gives rise to a homotopy co-orthogonal pair, and hence it is equivalent to a cellularization with respect to a set of objects. This question is not formally dual to the one discussed in [2, since the opposite of a locally presentable category is usually no longer locally presentable.

If one considers an arbitrary locally presentable category as a model category with weak equivalences being isomorphisms, and all morphisms being fibrations and cofibrations, then our result applies and seems to be new even in this case: under Vopěnka's principle any co-orthogonality class is generated by a set of objects.

A counterexample to a similar question for orthogonality classes in locally presentable categories was constracted in 4]. More specifically: it was shown that there are orthogonality classes in the category of groups which are not generated by any set of groups.

Additional motivation for the work on this paper was provided by Emmanuel Farjoun who asked: whether or not any continuous, augmented, homotopy idempotent functor from pointed simplicial sets to pointed simplicial sets is equivalent, in a certain set-theoretical framework, to a cellularization with respect to some simplicial set. This question is motivated, in turn, by the following construction: given a nullification with respect to a simplicial set $A$, the homotopy fibre $\bar{P}_{A}(X)$ of the natural map $X \rightarrow P_{A}(X)$ is a homotopy idempotent augmented functor, which is usually not equivalent to $\mathrm{CW}_{A} X$. We prove in this paper that there exist a simplicial set $B$ such that $\bar{P}_{A}(X)$ is weakly equivalent to $\mathrm{CW}_{B}(X)$ for all $X$. An alternative construction of $B$, not relying on Vopěnka's principle, was given in [6]

\section{Simplicial orthogonality AND CO-ORThogonality}

We will assume throughout the paper that $\mathcal{M}$ is a simplicial model category, but all the concepts in this section may be generalized to arbitrary model categories using homotopy function complexes. For every $A, X \in \mathcal{M}$ let $\tilde{A}$ denote the cofibrant replacement of $A$ and let $\hat{X}$ denote the fibrant replacement of $X$. 
We say that an object $X$ and a map $f: A \rightarrow B$ are homotopy orthogonal or simplicially orthogonal, $f_{\perp} \mathrm{h}_{\perp}$, if the induced map of simplicial sets

$$
f^{*}: \operatorname{map}(\tilde{B}, \hat{X}) \longrightarrow \operatorname{map}(\tilde{A}, \hat{X})
$$

is a weak equivalence.

Co-orthogonality is the dual concept: an object $A$ and a map $f: X \rightarrow Y$ are homotopy co-orthogonal, $A_{\mathrm{h}} \top f$ if the induced map of simplicial sets

$$
f_{*}: \operatorname{map}(\tilde{A}, \hat{X}) \longrightarrow \operatorname{map}(\tilde{A}, \hat{Y})
$$

is a weak equivalence.

More generally, if $\mathcal{S}$ is any class of maps, we denote by $\mathcal{S}^{\mathrm{h}} \perp$ the class of objects that are homotopy orthogonal to all the maps in $\mathcal{S}$, and say that objects in $\mathcal{S}^{\mathrm{h} \perp}$ are $\mathcal{S}$-local. The homotopy orthogonal complement of a class $\mathcal{D}$ of objects is defined similarly. Fibrant elements of $f^{\mathrm{h} \perp}$ are usually called $f$-local.

For a class of morphisms $\mathcal{S}$ in a model category $\mathcal{M}$, an $\mathcal{S}$-localization is a homotopy endofunctor $L: \mathcal{M} \rightarrow \mathcal{M}$ equipped with a natural transformation $\eta: \operatorname{Id} \rightarrow$ $L$ such that $\eta L \simeq L \eta$ and $L \eta: L \rightarrow L L$ is a weak equivalence on all objects, $\eta_{X}: X \rightarrow L X$ is in $\left(\mathcal{S}^{\mathrm{h}_{\perp}}\right)^{\mathrm{h} \perp}$ and $L X \in \mathcal{S}^{\mathrm{h} \perp}$ for all $X$ in $\mathcal{M}$. We also call it a localization with respect to $\mathcal{S}$ or, generically, a homotopy localization.

For a class of objects $\mathcal{A}$ in a model category $\mathcal{N}$, an $\mathcal{A}$-co-localization or $\mathcal{A}$ cellularization is a homotopy endofunctor $C: \mathcal{M} \rightarrow \mathcal{M}$ equipped with a natural transformation $\varepsilon: C \rightarrow \mathrm{Id}$ such that $\varepsilon C \simeq C \varepsilon$ and $C \varepsilon: C C \rightarrow C$ is a weak equivalence on all objects, $\varepsilon_{X}: C X \rightarrow X$ is in $\mathcal{A}^{\mathrm{h}}$ and $C X \in\left(\mathcal{A}^{\mathrm{T}}{ }^{\mathrm{T}}\right)^{\mathrm{h}}$ for all $X$ in $\mathcal{M}$. We also call it a co-localization with respect to $\mathcal{A}$ or, generically, a homotopy cellularization.

Recall that a partially ordered set $P$ is called $\lambda$-directed, where $\lambda$ is a regular cardinal, if every subset of $P$ of cardinality smaller than $\lambda$ has an upper bound.

Lemma 1.1. Given a cofibrantly generated simplicial model category $\mathcal{M}$ and a small category $\mathcal{C}$, consider the projective simplicial model structure on the category of functors $\mathcal{M}^{\mathcal{C}}$ described in [10, Theorem 11.7.3]. Suppose that $A$ is a cofibrant diagram in this model structure and $X$ is a fibrant object of $\mathcal{M}$, then the $\mathcal{C}^{\mathrm{op}}$ diagram $\operatorname{hom}(A, X)$ of simplicial sets is fibrant in the injective model structure on $\mathcal{S}^{\mathrm{eop}}$.

Proof. We have to show that any commutative square

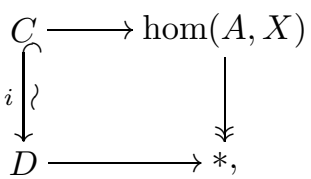

where $i$ is an injective (objectwise) trivial cofibration of $\mathcal{C}^{\text {op }}$-diagrams of simplicial sets, admits a lift. By adjunction this problem is equivalent to finding a lift in the following commutative square in $\mathcal{M}$ :

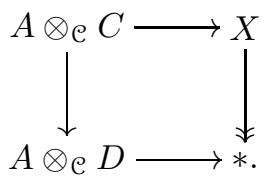


And this problem is equivalent, by another adjunction, to finding a lift in the following commutative square in $\mathcal{M}^{\mathrm{e}}$ :

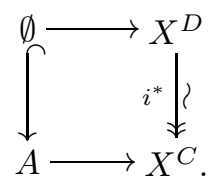

In the last square the lift exists, since $A$ is projectively cofibrant and $i^{*}$ is an objectwise trivial fibration, i.e., projective fibration.

Lemma 1.2. Let $\mathcal{S}$ be any class of morphisms in a combinatorial simplicial model category $\mathcal{M}$, and let $\mathcal{S}^{\mathrm{T}}=\mathcal{A}$ be its homotopy co-orthogonal complement. Then there exists a regular cardinal $\lambda$ such that $\mathcal{A}$ is closed under $\lambda$-directed colimits in $\mathcal{M}$.

Proof. Let $I$ be a set of generating cofibrations for the model category $\mathcal{M}$. Choose a regular cardinal $\lambda$ such that any object of the set of domains and codomains of maps in $I$ is $\lambda$-presentable (such a cardinal exists since the category $\mathcal{M}$ is locally presentable). Let $P$ be any $\lambda$-directed partially ordered set, and suppose given a diagram $A: P \rightarrow \mathcal{M}$. Let us depict it, for simplicity, as a chain:

$$
A_{0} \longrightarrow A_{1} \longrightarrow \cdots \longrightarrow A_{p} \longrightarrow \cdots
$$

Suppose that the objects $A_{p}$ are in $\mathcal{A}$ for each $p \in P$. We need to show that the colimit of this diagram is also in $\mathcal{A}$.

Consider the category $\mathcal{M}^{P}$ of $P$-indexed diagrams in $\mathcal{M}$, and endow it with a model structure as described in [10,11.6]. Thus, weak equivalences and fibrations are objectwise, and cofibrations are retracts of free cell complexes. The diagram $A$ may be viewed as a single element in $\mathcal{M}^{P}$. Let $\tilde{A}$ be a cofibrant approximation of $A$ in the above model structure, hence obtaining the following commutative diagram in $\mathcal{M}$ :

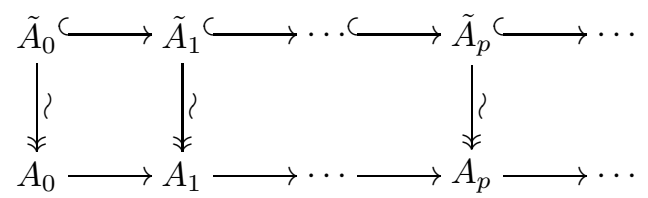

where $\tilde{A}_{p}$ is a cofibrant approximation of $A_{p}$ in $\mathcal{M}$.

Note that $\operatorname{colim} \tilde{A}_{p}$ is a cofibrant object in $\mathcal{M}$, since $\operatorname{colim} \mathcal{M}^{P} \rightarrow \mathcal{M}$ is a left Quillen functor [10, 11.6.8], hence preserves cofibrations.

For every map $f: X \rightarrow Y$ in $\mathcal{S}$, let $\hat{f}: \hat{X} \rightarrow \hat{Y}$ be a fibrant approximation to $f$. The induced map

$$
\operatorname{map}\left(\operatorname{colim} \tilde{A}_{p}, \hat{f}\right): \operatorname{map}\left(\operatorname{colim} \tilde{A}_{p}, \hat{X}\right) \longrightarrow \operatorname{map}\left(\operatorname{colim} \tilde{A}_{p}, \hat{Y}\right)
$$

can be written as

$$
\lim \operatorname{map}\left(\tilde{A}_{p}, \hat{f}\right): \lim \operatorname{map}\left(\tilde{A}_{p}, \hat{X}\right) \longrightarrow \lim \operatorname{map}\left(\tilde{A}_{p}, \hat{Y}\right) .
$$

By Lemma 1.1 each of the $P^{\text {op }}$-diagrams of simplicial sets map $(\tilde{A}, \hat{X})$ and $\operatorname{map}(\tilde{A}, \hat{Y})$ is a fibrant object in the injective model structure on the category of $P^{\mathrm{op}}$-diagrams of simplicial sets $\mathcal{S}^{P^{\mathrm{op}}}$, since $\tilde{A}$ is a cofibrant diagram in the projective model structure on $\mathcal{M}^{P^{\text {op }}}$ and $\hat{X}, \hat{Y}$ are fibrant. Therefore, map $\left(\operatorname{colim} \tilde{A}_{p}, \hat{f}\right)$ 
is a homotopy inverse limit of weak equivalences, so it is itself a weak equivalence. This shows that $\operatorname{colim} \tilde{A}_{p}$ is in $\mathcal{A}$.

Trivial fibrations in $\mathcal{M}$ are preserved under $\lambda$-directed colimits, since the set of generating cofibrations has $\lambda$-presentable domains and codomains, hence there is a trivial fibration

$$
\operatorname{colim} \tilde{A}_{p} \stackrel{\sim}{\longrightarrow} \operatorname{colim} A_{p}
$$

We conclude that colim $\tilde{A}_{p}$ is a cofibrant approximation of the colim $A_{p}$, since any directed colimit of a projectively cofibrant diagram is cofibrant $(\tilde{A}$ is a cofibrant diagram in $\mathcal{M}^{A}$ and the colimit functor $\mathcal{M}^{A} \rightarrow \mathcal{M}$ is left Quillen by [10 11.6.8(1)]). Hence, colim $A_{p}$ is in $\mathcal{A}$, as claimed.

The next lemma significantly relies on Vopěnka's principle [1].

Lemma 1.3. Suppose that Vopěnka's principle is true. Let $\mathcal{S}$ be any class of morphisms in a combinatorial simplicial model category $\mathcal{M}$, and let $\mathcal{D}=\mathcal{S}^{\mathrm{n}}{ }^{\top}$. Then there exists a set of objects $\mathcal{X}$ such that $\mathcal{X}^{\mathrm{h}^{\top}}=\mathcal{D}^{\mathrm{h}^{\top}}$.

Proof. By abuse of notation, we also denote by $\mathcal{D}$ the full subcategory of $M$ generated by the class $\mathcal{D}$. Since $\mathcal{M}$ is locally presentable, assuming Vopěnka's principle, it follows from [1 Theorem 6.6] that $\mathcal{D}$ is bounded, i.e., it has a small dense subcategory. We have shown in Lemma 1.2 that there exists a regular cardinal $\lambda$ such that $\mathcal{D}$ is closed under $\lambda$-directed colimits. Hence, by [1, Corollary 6.18], the full subcategory generated by $\mathcal{D}$ in $M$ is accessible. Thus, for a certain regular cardinal $\lambda_{0} \geq \lambda$, the class $\mathcal{D}$ contains a set $\mathcal{X}$ of $\lambda_{0}$-presentable objects such that every object of $\mathcal{D}$ is a $\lambda_{0}$-directed colimit of objects of $\mathcal{X}$.

Since $\mathcal{X} \subset \mathcal{D}$, we have $\mathcal{X}^{\mathrm{h}^{\top}} \supset \mathcal{D}^{\mathrm{h}^{\top}}$ and $\left(\mathcal{X}^{\mathrm{h}^{\top}}\right)^{\mathrm{h}^{\top}} \subset\left(\mathcal{D}^{\mathrm{h}^{\top}}\right)^{\mathrm{h}^{\top}}=\mathcal{S}$. Our aim now is to show the reverse inclusion $\left(\mathcal{X}^{\mathrm{h}^{\top}}\right)^{\mathrm{h}^{\top}} \supset \mathcal{S}$. By Lemma 1.2 $\left(\mathcal{X}^{\mathrm{h}^{\top}}\right)^{\mathrm{h}^{\top}}$ is closed under $\lambda$-directed colimits. Hence $\left(\mathcal{X}^{\mathrm{h}^{\top}}\right)^{\mathrm{h}}$ is also closed under $\lambda_{0}$-directed colimits and every element of $\mathcal{D}$ is a $\lambda_{0}$-directed colimit of elements of $\mathcal{X}$. Then we can choose $\mathcal{X}$ as our generating set.

Theorem 1.4. Let $\mathcal{M}$ be a right proper, combinatorial, simplicial model category. If Vopěnka's principle is assumed true, then for any (possibly proper) class of objects $\mathcal{D}$ there is a cellularization functor $C W_{\mathcal{D}}$ with respect to $\mathcal{D}$.

Proof. By Lemma 1.3 there exists a set $\mathcal{X}$ of objects in $\mathcal{M}$ such that $\mathcal{X}^{\mathrm{h}^{\top}}=\mathcal{D}^{\mathrm{h}^{\top}}$. Then the cellularization with respect to this set $\mathcal{X}$ has $\mathcal{D}^{\mathrm{h}}$ as its class of co-local equivalences, i.e. it is equivalent to the cellularization with respect to $\mathcal{D}$. Under the assumptions of the theorem, the existence of the cellularization functor with respect to a set of objects was established in [10.

\section{Simplicial Idempotent FunCtors and Simplicial CO-ORTHOGONALity}

In the proof of the main theorem bellow we rely on results, which are dual to some of the statements in 8 . The assumption of simpliciality (on functors) may be removed (if required) similarly to [2].

Theorem 2.1. Let $\mathcal{M}$ be a cofibrantly generated simplicial model category. Let $C$ be an augmented, homotopy idempotent simplicial functor from $\mathcal{M}$ to $\mathcal{M}$. Then, assuming Vopěnka's principle, $C$ is equivalent to homotopy cellularization with respect to some set of objects. 
Proof. Formally, homotopy cellularization is a homotopy localization in the opposite category. The argument of $[8]$ generalizes to any simplicial model category and continuous localization functor, since it does not use any small-object considerations. It shows that any simplicial localization functor is a localization with respect to the class $\mathcal{S}$ of maps which are rendered into equivalences by the localization

functor. Or, equivalently, this is a localization with respect to the class $\mathcal{D}=\mathcal{S}^{\mathrm{h}} \perp$ of objects. In other words, the initial functor $C$ is a cellularization with respect to $\mathcal{D}$.

By Lemma 1.3 there exists a set $\mathcal{X}$ of elements such that $\mathcal{D}^{{ }^{\top}}=\mathcal{X}^{{ }^{\top}}$. Therefore, the cellularization with respect to $\mathcal{X}$ is equivalent to $C$.

\section{REFERENCES}

[1] J. Adámek and J. Rosický. Locally Presentable and Accessible Categories. London Math. Soc. Lecture Note Ser. 189. Cambridge University Press, Cambridge, 1994.

[2] C. Casacuberta and B. Chorny. The orthogonal subcategory problem in homotopy theory. In D. Arlettaz and K. Hess, editors, An Alpine Anthology of Homotopy Theory, volume 399 of Contemporary Mathematics, pages 41-53, Providence, Rhode Island, 2006. AMS.

[3] C. Casacuberta, A. Frei, and G. C. Tan. Extending localization functors. J. Pure Appl. Algebra, 103(2):149-165, 1995.

[4] C. Casacuberta and D. Scevenels. On the existence of group localizations under large-cardinal axioms. RACSAM Rev. R. Acad. Cienc. Exactas Fís. Nat. Ser. A Mat., 95(2):163-170, 2001.

[5] C. Casacuberta, D. Scevenels, and J. H. Smith. Implications of large-cardinal principles in homotopical localization. Adv. Math., 197(1):120-139, 2005.

[6] W. Chachólski, P.-E. Parent, and D. Stanley. Cellular generators. Proc. Amer. Math. Soc., 132(11):3397-3409 (electronic), 2004.

[7] B. Chorny. Localization with respect to a class of maps. II. Equivariant cellularization and its application. Israel J. Math., 147:141-155, 2005.

[8] E. Dror Farjoun. Higher homotopies of natural constructions. J. Pure Appl. Algebra, 108(1):23-34, 1996.

[9] D. Dugger. Combinatorial model categories have presentations. Adv. Math., 164(1):177-201, 2001.

[10] P. S. Hirschhorn. Model categories and their localizations, volume 99 of Mathematical Surveys and Monographs. American Mathematical Society, Providence, RI, 2003.

[11] J. Rosický and W. Tholen. Left-determined model categories and universal homotopy theories. Trans. Amer. Math. Soc., 355(9):3611-3623 (electronic), 2003.

Department of Mathematics, ETh Zentrum, 8092 Zurich, Switzerland

E-mail address: chorny@math.ethz.ch 